\title{
AUTOMORPHISMS OF A FREE GROUP OF INFINITE RANK
}

\author{
C. K. GUPTA AND W. HOŁUBOWSKI
}

\begin{abstract}
The problem of classifying the automorphisms of a free group of infinite countable rank is investigated. Quite a reasonable generating set for the group Aut $F_{\infty}$ is described. Some new subgroups of this group and structural results for them are presented. The main result says that the group of all automorphisms is generated (modulo the $I A$-automorphisms) by strings and lower triangular automorphisms.
\end{abstract}

\section{$\S 1$. INTRODUCTION}

The automorphism group of a free group of finite rank has been investigated intensively. In [24], Nielsen obtained its presentation in terms of "elementary" automorphisms, which are called the Nielsen automorphisms nowadays. His powerful method initiated extensive research and systematic study in this area. Nice surveys of the results can be found in [22] and [19].

Unlike the case of finite rank, the situation for the automorphism group Aut $F_{\infty}$ of a free group $F_{\infty}$ of infinite countable rank is not well understood. The problem of classification of all its subgroups seems to be very difficult. Only a few natural subgroups and isolated results are known. The group Aut $F_{\infty}$ is "very big"; for example, the group $\operatorname{Sym}(\mathbb{N})$ of all permutations on the natural numbers can be viewed as its subgroup. Moreover, since $\operatorname{Sym}(\mathbb{N})$ is known to contain a free group of rank $2^{\aleph_{0}}$ [11], we obtain one more subgroup of Aut $F_{\infty}$.

Some nice properties of Aut $F_{\infty}$ are related to the properties of the free group $F_{\infty}$ on free generators $x_{1}, x_{2}, \ldots$ For example, $F_{\infty}$ has the basis cofinality property [19]; i.e., for every $\alpha \in$ Aut $F_{\infty}$ and every $n \in \mathbb{N}$ there exists $r \in \mathbb{N}$ and $\beta \in \operatorname{Aut}\left(\left\langle x_{1}, \ldots, x_{r}\right\rangle\right)$ such that $r \geq n$ and $\beta\left(x_{i}\right)=\alpha\left(x_{i}\right)$ for $i=1, \ldots, n$ (here $\left\langle x_{1}, \ldots, x_{r}\right\rangle$ denotes the subgroup generated by $\left.x_{1}, \ldots, x_{r}\right)$. The group $F_{\infty}$ has also the small index property; i.e., for every subgroup $\Lambda$ in Aut $F_{\infty}$ of index less than $2^{\aleph_{0}}$ there exists a finite subset $Y$ of $F_{\infty}$ such that the pointwise stabilizer of $Y$ in Aut $F_{\infty}$ is contained in $\Lambda$ (the converse statement is obvious). In particular, these results imply that Aut $F_{\infty}$ is not the union of an ascending chain of countably many proper subgroups [1].

The subgroup lattice of Aut $F_{\infty}$ is far from being understood. Some natural subgroups consisting of inner, finitary, bounded, triangular, permutational, or diagonal automorphisms have been studied (the definitions are given in the next section). However, even for the group of bounded automorphisms no satisfying generating set is known. In [9], Solitar conjectured that this group can be generated by infinite elementary simultaneous

2000 Mathematics Subject Classification. Primary 20 E05.

Key words and phrases. Free group of infinite rank, automorphism group, string. 
Nielsen automorphisms, but this problem still remains open. We note that, in the infinite case, the usual Nielsen automorphisms generate the group Aut $_{\text {fin }} F_{\infty}$ consisting of all automorphisms that act nontrivially only on finitely many generators (see [19]).

In our paper we construct some new subgroups of Aut $F_{\infty}$ and give structural results for them.

The standard notion of boundedness for an automorphism $\alpha \in$ Aut $F_{\infty}$ requires a fixed upper bound $n$ on the length of each freely reduced word of the form $\alpha\left(x_{i}\right)$ or $\alpha^{-1}\left(x_{i}\right)$. We shall consider boundedness from a different point of view. We require only that each $x_{j}$ occurring in $\alpha\left(x_{i}\right)$ be "not far" from $x_{i}$ in the sense of $|i-j|$ being sufficiently small. Note that the exponent sum of $x_{j}$ in $\alpha\left(x_{i}\right)$ can be arbitrary. It is very far from the notion of boundedness introduced in [9]. In $\S 2$ we also introduce a notion of a string, which realizes this idea. The strings are analogs of infinite block-diagonal matrices with an infinite number of finite blocks on the main diagonal. A special kind of strings in the group of upper triangular automorphisms was considered in [9]. Our fundamental observation is that the set of finite compositions of strings forms a group $\mathcal{H}$, called the group of strings. This group plays a crucial role in our considerations. In $\S 3$ we prove that $\mathcal{H}$ contains the group generated by the permutational and the upper triangular automorphisms and investigate some properties of parabolic subgroups of $\mathcal{H}$. We also describe a large sublattice of subgroups of $\mathcal{H}$ associated with growths. The main result in $\S 4$ says that Aut $F_{\infty}$ is generated (modulo the $I A$-automorphisms) by strings and lower triangular automorphisms. In the last section we formulate similar results for automorphisms of relatively free groups for some varieties of groups.

Our results remain valid also for a free group of uncountable rank. However, we need to assume that the set of free generators is well ordered. This condition is not surprising, since even the proof of the Nielsen-Schreier theorem (saying that every subgroup of a free group is free) requires such an ordering in the case of infinite rank (see [7, p. 89]).

\section{§2. Definitions And EXAmples}

Let $F_{\infty}$ be the free group on a countable set of free generators $X=\left\{x_{i}, i \in \mathbb{N}\right\}$, and let Aut $F_{\infty}$ be the group of its automorphisms. Let $\alpha \in \operatorname{Aut} F_{\infty}$. We say that an automorphism $\alpha$ is

upper triangular if $\alpha\left(x_{i}\right) \in\left\langle x_{i+k}: k \in \mathbb{N} \cup\{0\}\right\rangle$ for all $i \in \mathbb{N}$;

lower triangular if $\alpha\left(x_{1}\right)=x_{1}^{ \pm 1}$ and $\alpha\left(x_{i}\right) \in\left\langle x_{1}, \ldots, x_{i}\right\rangle$ for all $i>1$;

diagonal if $\alpha\left(x_{i}\right)=x_{i}^{\epsilon_{i}}$ for $\epsilon_{i} \in\{1,-1\}$ and all $i \in \mathbb{N}$;

a permutational automorphism if $\alpha\left(x_{i}\right)=x_{\pi(i)}$ for some fixed permutation $\pi$ on the natural numbers;

a column-finite automorphism if for every $i \in \mathbb{N}, x_{i}$ occurs only in a finite number of freely reduced words $\alpha\left(x_{j}\right), j \in \mathbb{N}$.

The following results of $\mathrm{R}$. Cohen characterize the upper and lower triangular automorphisms.

Lemma 1 [9, Theorem 2.4]. If an automorphism $\alpha$ is upper triangular, then, for every $i=1,2, \ldots,\left.\alpha\right|_{\left\langle x_{i}, x_{i+1}, \ldots\right\rangle}$ is an automorphism of $\left\langle x_{i}, x_{i+1}, \ldots\right\rangle$ and $\alpha\left(x_{i}\right)=A_{i} x_{i}^{\epsilon_{i}} B_{i}$, where $\epsilon_{i} \in\{1,-1\}$ and $A_{i}, B_{i} \in\left\langle x_{i+1}, x_{i+2}, \ldots\right\rangle$.

Lemma 2 [9, Corollary 2.3]. If an automorphism $\alpha$ is lower triangular, then, for every $i=1,2, \ldots, \alpha\left(x_{i}\right)=A_{i} x_{i}^{\epsilon_{i}} B_{i}$, where $\epsilon_{i} \in\{1,-1\}, A_{1}=B_{1}=1$, and $A_{i}, B_{i} \in$ $\left\langle x_{1}, \ldots, x_{i-1}\right\rangle$ for $i>1$.

We say that an automorphism $\alpha \in$ Aut $F_{\infty}$ is $n$-bounded if the words $\alpha\left(x_{i}\right)$ and $\alpha^{-1}\left(x_{i}\right)$ have length at most $n$ for all $i$, and $\alpha$ is bounded if $\alpha$ is $n$-bounded for some $n$. 
Let $\mathcal{B}_{n}$ denote the set of all $n$-bounded automorphisms, and let $\mathcal{B}=\bigcup_{n=1}^{\infty} \mathcal{B}_{n}$. Clearly, $\mathcal{B}_{n} \subseteq \mathcal{B}_{n+1}$ for all $n$.

Let $\mathcal{T}^{+}, \mathcal{T}^{-}, \mathcal{D}, \mathcal{S}$ denote the sets of all upper triangular, lower triangular, diagonal, and permutational automorphisms, respectively, and let $\mathcal{K}$ be the set of all automorphisms $\alpha$ such that both $\alpha$ and $\alpha^{-1}$ are column-finite. Clearly, $\mathcal{D}, \mathcal{S} \subseteq \mathcal{B}_{1}, \mathcal{T}^{+} \cap \mathcal{T}^{-}=\mathcal{D}$, and the intersections $\mathcal{T}^{+} \cap \mathcal{S}$ and $\mathcal{T}^{-} \cap \mathcal{S}$ are trivial. From now on we shall not distinguish a permutational automorphism and the corresponding permutation on the natural numbers.

The following result describes some natural subgroups of Aut $F_{\infty}$.

Proposition 1. The sets $\mathcal{T}^{+}, \mathcal{T}^{-}, \mathcal{D}, \mathcal{S}, \mathcal{K}$, and $\mathcal{B}$ form subgroups of Aut $F_{\infty}$.

Proof. For $\mathcal{D}$ and $\mathcal{S}$, the statement is obvious. Since both $\mathcal{K}$ and $\mathcal{B}$ are closed under taking compositions, they are subgroups of Aut $F_{\infty}$. The results for $\mathcal{T}^{+}$and $\mathcal{T}^{-}$can be deduced from Lemmas 1 and 2 .

Remark. $\mathcal{B}$ is called the group of bounded automorphisms. By analogy with infinite matrices, we call $\mathcal{K}$ the group of column-finite automorphisms.

The group $\mathcal{S}$ contains a free subgroup of rank $2^{\aleph_{0}}$ (see [11]). However, for $\mathcal{T}^{+}$we can only prove the following statement.

Theorem 1. The group $\mathcal{T}^{+}$contains a free subgroup of countable rank.

Proof. Let $\alpha_{1}, \alpha_{2}$ be two automorphisms of $F_{\infty}$ defined as follows: for all $k \in \mathbb{N}$,

$\alpha_{1}\left(x_{2 k-1}\right)=x_{2 k-1} x_{2 k}, \quad \alpha_{1}\left(x_{2 k}\right)=x_{2 k}$,

$\alpha_{2}\left(x_{2 k-1}\right)=x_{2 k-1}, \quad \alpha_{2}\left(x_{2 k}\right)=x_{2 k} x_{2 k+1}$.

Let $A_{\infty}$ denote a free Abelian group of countable rank, and let $\chi:$ Aut $F_{\infty} \rightarrow$ Aut $A_{\infty}$ be the homomorphism induced by the natural mapping $\bar{\chi}: F_{\infty} \rightarrow A_{\infty}$. Since Aut $A_{\infty}$ is isomorphic to $G L(\infty, \mathbb{Z})$ (this is the group of invertible row-finite countably infinite matrices over $\mathbb{Z}$ ), we shall identify $\chi(f)$ with the corresponding matrix in $G L(\infty, \mathbb{Z})$. In [16] it was proved that the matrices $\chi\left(\alpha_{1}\right), \chi\left(\alpha_{2}\right)$ generate a free subgroup of rank two in $G L(\infty, \mathbb{Z})$. This means that $\left\langle\alpha_{1}, \alpha_{2}\right\rangle$ is also a free group of rank two contained in $\mathcal{T}^{+} \cap \mathcal{B}$. Its commutator subgroup is a required free group of countable rank.

Now we define a notion fundamental to our paper.

Definition 1. Let $\alpha$ be an automorphism of $F_{\infty}$. If the following two conditions are fulfilled:

(1) there is a partition $\left\{X_{j} \mid j \in \mathbb{N}\right\}$ of the set of generators $\left\{x_{1}, x_{2}, \ldots\right\}$ such that $X_{1}=\left\{x_{1}, \ldots, x_{n_{1}}\right\}$ and for $j>1$ we have $X_{j}=\left\{x_{n_{j}+1}, \ldots, x_{n_{j+1}}\right\}$, where $n_{1}<n_{2}<\cdots$ is a strictly increasing sequence of natural numbers;

(2) for $n_{j}+1 \leq k \leq n_{j+1}$ we have $\alpha\left(x_{k}\right) \in\left\langle x_{n_{j}+1}, \ldots, x_{n_{j+1}}\right\rangle$, then $\alpha$ will be called a string.

Note that if $\alpha$ is a string, we can write $F_{\infty}=\prod_{j \in \mathbb{N}}^{\star}\left\langle X_{j}\right\rangle$, where ${ }^{\star}$ denotes a free product, and define automorphisms $\alpha_{j}$ to act as $\alpha$ on $\left\langle X_{j}\right\rangle$ (this means that $\alpha_{j}=\left.\alpha\right|_{\left\langle X_{j}\right\rangle}$ on $\left.\left\langle X_{j}\right\rangle\right)$ and to act trivially on $\left\langle X_{i}\right\rangle$ with $i \neq j$. Thus, $\alpha$ can be expressed as a product, $\alpha=\prod_{j=1}^{\infty} \alpha_{j}$, and there is no ambiguity in this representation, because the $\alpha_{j}$ commute and all but a finite number of these automorphisms act trivially on each particular freely reduced word in $F_{\infty}$.

If $\alpha$ is a string and $\alpha=\prod \alpha_{j}$, then $\alpha^{-1}$ is also a string (because $\alpha^{-1}=\prod \alpha_{j}^{-1}$ ). However, a composition of two strings may fail to be a string, as the following example shows. 
Example. Let $\alpha_{1}$ and $\alpha_{2}$ be defined as in the proof of Theorem 1. Then $\alpha_{1}, \alpha_{2}$ are strings and $\alpha_{2} \circ \alpha_{1}$ is not a string, because $\alpha_{2} \circ \alpha_{1}\left(x_{2 k-1}\right)=x_{2 k-1} x_{2 k}$ and $\alpha_{2} \circ \alpha_{1}\left(x_{2 k}\right)=$ $x_{2 k} x_{2 k+1} x_{2 k+2}$ for all $k$.

Remark. In [9], a string in $\mathcal{T}^{+}$was called a splitting automorphism.

Let $\mathcal{H}$ be the set of all finite compositions of strings (for various partitions, in general). The following observation is clear, but we state it explicitly because of its great importance.

Proposition 2. The set $\mathcal{H}$ is a subgroup of Aut $F_{\infty}$.

The group $\mathcal{H}$, which we call the group of strings, plays a crucial role in further considerations.

Example. Let $\beta\left(x_{1}\right)=x_{1}$ and $\beta\left(x_{k}\right)=x_{1} \cdot x_{k}$ for all $k>1$. Clearly, $\beta$ is an automorphism belonging to $\mathcal{T}^{-} \cap \mathcal{B}$, and $\beta \notin \mathcal{H}$.

\section{§3. The structure of The Group of STRINGS}

In this section we consider the subgroup structure of the group $\mathcal{H}$ of strings.

Proposition 3. The group $\mathcal{H}$ contains the subgroup generated by the upper triangular and the permutational automorphisms, i.e., $\mathcal{H} \supseteq\left\langle\mathcal{T}^{+}, \mathcal{S}\right\rangle$.

Proof. The inclusion $\mathcal{T}^{+} \subseteq \mathcal{H}$ follows from Theorem 3.3 in [9]. Lemma 2.3 in [27] (see also [25]) implies that $\mathcal{S} \subseteq \mathcal{H}$, which finishes the proof.

It seems plausible that $\mathcal{H}=\left\langle\mathcal{T}^{+}, \mathcal{S}\right\rangle$; however, neither a proof nor a counterexample is known.

The above result on the structure of the group $\mathcal{H}$ can be used for describing its subgroups. We say that a subgroup $G$ of $\mathcal{H}$ is parabolic if it contains $\mathcal{T}^{+}$. It is clear that the description of the parabolic subgroups in $\mathcal{H}$ depends heavily on the subgroup structure of the symmetric group $\operatorname{Sym}(\mathbb{N})$. Moreover, for every subgroup $S_{1}$ of $\mathcal{S}$ the subgroup $\left\langle\mathcal{T}^{+}, S_{1}\right\rangle$ is parabolic. If $S_{2}$ is a proper subgroup of $S_{1}$, then $\left\langle S_{2}, \mathcal{T}^{+}\right\rangle$is a proper subgroup of $\left\langle S_{1}, \mathcal{T}^{+}\right\rangle$. Therefore, it seems that the minimal parabolic subgroups in $\mathcal{H}$ can be found by using such arguments. A more detailed discussion on this topic will appear elsewhere.

Two subgroups $A, B$ are said to be comparable if $A \subseteq B$ or $B \subseteq A$, and incomparable otherwise. The known properties of subgroups in $S(\mathbb{N})$ imply the following statement.

Theorem 2. There exists an uncountable family of pairwise incomparable parabolic subgroups in $\mathcal{H}$.

Proof. First, we recall a classical result of W. Sierpiński, proved in 1928 (see [26]). A subset $\Gamma$ of an infinite set $\Delta$ is called a moiety if $|\Gamma|=|\Delta \backslash \Gamma|$. Let $\Delta=\mathbb{Q}$. For each real number $r$, we can take an infinite sequence $\Gamma_{r}$ of distinct rationals that converges to $r$. Then the set $\left\{\Gamma_{r}: r \in \mathbb{R}\right\}$ forms an uncountable family of moieties such that, for any two distinct members $\Gamma, \Gamma^{\prime}$ of this family, the set $\Gamma \cap \Gamma^{\prime}$ is finite.

Now, let $\phi: \mathbb{Q} \rightarrow \mathbb{N}$ be any bijection, and let $\bar{\Gamma}_{r}:=\phi\left(\Gamma_{r}\right)$. We denote by $S\left(\bar{\Gamma}_{r}\right)$ the pointwise stabilizer of the set $\mathbb{N} \backslash \bar{\Gamma}_{r}$ in $S(\mathbb{N})$. It is easily seen that the family $\left\{\left\langle S\left(\bar{\Gamma}_{r}\right), \mathcal{T}^{+}\right\rangle: r \in \mathbb{R}\right\}$ consists of pairwise incomparable parabolic subgroups.

In other words, the above theorem states that a lattice of parabolic subgroups of $\mathcal{H}$ contains an uncountable family of chains.

To describe another large family of subgroups of $\mathcal{H}$, we need the notion of a growth. Let $\mathbb{N}$ be the set of positive integers with natural order. We extend this order to the set 
$\mathbb{N}_{\infty}:=\mathbb{N} \cup\{\infty\}$, assuming that $n<\infty$ for all $n \in \mathbb{N}$. The set $P(\mathbb{N})$ of all functions $f$ : $\mathbb{N}_{\infty} \rightarrow \mathbb{N}_{\infty}$, equipped with the operation of composition of functions, forms a semigroup. We denote by $\Omega_{\infty}$ the subsemigroup of all functions $f \in P(\mathbb{N})$ satisfying the conditions $f(\infty)=\infty$ and $f(n+1) \geq f(n)>n$ for all $n \in \mathbb{N}$. We introduce an order on $\Omega_{\infty}$ : for every $f, g \in \Omega_{\infty}$ we write

$f \prec g$ if and only if there exists $n_{0}$ such that $f(n)<g(n)$ for all $n>n_{0}$.

As usual, $f<g$ means that $f(n)<g(n)$ for all natural $n$.

We write $f \ll g$ if for all $k \in \mathbb{N}$ we have $f^{k} \prec g$, where $f^{k}$ denotes the composition of $k$ copies of $f$. We take $M(f):=\left\{h \in \Omega_{\infty}: f \ll h\right\}$ and define an equivalence relation $\sim$ on $\Omega_{\infty}$ :

$$
f \sim g \quad \text { if and only if } M(f)=M(g) .
$$

Let $\Omega^{\star}$ denote the set of equivalence classes of $\sim$. Each element of $\Omega^{\star}$ is called a growth.

For example, consider two special functions in $\Omega_{\infty}: \quad f_{\infty}(n)=\infty$ and $f_{0}(n)=n+1$; their growths are $\omega_{\infty}=\left[f_{\infty}\right]$ and $\omega_{0}=\left[f_{0}\right]$. Clearly, $f \in \omega_{\infty}$ if there exists $n \in \mathbb{N}$ such that $f(n)=\infty$, and $g \in \omega_{0}$ if there exists $d \in \mathbb{N}$ such that $g(n) \leq n+d$ for all $n \in \mathbb{N}$.

We define a partial order $\leq$ in $\Omega^{\star}$ :

$$
\omega_{1} \leq \omega_{2} \quad \text { if and only if } M(f) \supseteq M(g) \text { for some } f \in \omega_{1}, g \in \omega_{2} \text {. }
$$

Clearly, for all $\omega \in \Omega^{\star}$ we have $\omega_{0} \leq \omega \leq \omega_{\infty}$. If $\omega_{1}=[f]$ and $\omega_{2}=[g]$, then we can define two new growths:

$$
\begin{aligned}
\omega_{1} \vee \omega_{2} & =[\max \{f, g\}], \\
\omega_{1} \wedge \omega_{2} & =[\min \{f, g\}] .
\end{aligned}
$$

The main result of [17] reads as follows.

Theorem 3. Equipped with the operations $\omega_{1} \vee \omega_{2}$ and $\omega_{1} \wedge \omega_{2}$, the set $\Omega^{\star}$ forms a lattice with the following properties:

a) $\Omega^{\star}$ has a unique minimal element $\omega_{0}$ and a unique maximal element $\omega_{\infty}$;

b) $\Omega^{\star}$ is dense, i.e., if for some $\omega_{1}, \omega_{2} \in \Omega^{\star}$ we have $\omega_{1}<\omega_{2}$, then there exists $\omega_{3} \in \Omega^{\star}$ such that $\omega_{1}<\omega_{3}<\omega_{2}$;

c) $\Omega^{\star}$ has neither atoms nor coatoms;

d) for every $\omega$ such that $\omega_{0}<\omega<\omega_{\infty}$, there exists an uncountable family (an uncountable antichain) of pairwise incomparable growths that are not comparable with $\omega$;

e) $\Omega^{\star}$ is distributive and modular.

Now we define some subgroups of $\mathcal{H}$ associated with growths. Let $\alpha$ be a string corresponding to a sequence $n_{1}<n_{2}<\cdots$. We say that $\alpha$ is bounded by a function $f$ of class $\omega$ if $f(1) \geq n_{1}$ and $f\left(n_{k}+1\right) \geq n_{k+1}$ for each natural $k$. We say that an automorphism $\beta$ in Aut $F_{\infty}$ is bounded by $f \in \omega$ (or $\beta$ is $[f]$-bounded) if for every natural $n$ there exists $k=k(n)$ depending only on $n$ such that $\beta\left(x_{n}\right)$ and $\beta^{-1}\left(x_{n}\right)$ are reduced words in the variables $x_{n-k(n)}, \ldots, x_{n-1}, x_{n}, x_{n+1}, \ldots, x_{n+k(n)}$ and $f(n) \geq n+k(n)$.

It is easily seen that if $\alpha$ is $[f]$-bounded and $\beta$ is $[g]$-bounded, then $\alpha \circ \beta$ is $[f \circ g]$ bounded. Hence, the following definition makes sense.

Definition 2. We denote by $\mathcal{H}(\omega)$ the set of all automorphisms $\alpha \in \mathcal{H}$ that are $[f]$ bounded for some $f \in \omega$.

In fact, $\mathcal{H}(\omega)$ is a well-defined subgroup of $\mathcal{H}$.

Theorem 4. The family $\left\{\mathcal{H}(\omega) \mid \omega \in \Omega^{\star}\right\}$ forms a sublattice of the lattice of subgroups of $\mathcal{H}$. Moreover, this sublattice is isomorphic to the lattice $\Omega^{\star}$.

In particular, this means that the lattice $\left\{\mathcal{H}(\omega) \mid \omega \in \Omega^{\star}\right\}$ has all the properties listed in Theorem 3. 


\section{$\S 4$. The STruCture of Aut $F_{\infty}$}

In this section we prove some structural results for Aut $F_{\infty}$ and give a description of some normal subgroups. Let $A_{\infty} \cong F_{\infty} / F_{\infty}^{\prime}$ be a free Abelian group of infinite countable rank, and let $\chi:$ Aut $F_{\infty} \rightarrow$ Aut $A_{\infty}$ be the homomorphism induced by the natural map $\bar{\chi}: F_{\infty} \rightarrow A_{\infty}$. The kernel $\mathcal{A}$ of $\chi$ consists of the $I A$-automorphisms of Aut $F_{\infty}$. As was proved in [8] and [20], $\chi$ is an epimorphism. We shall use the known isomorphism from Aut $A_{\infty}$ onto $G L(\infty, \mathbb{Z})$, the group of invertible row-finite countably infinite matrices over $\mathbb{Z}$. We shall identify $\chi(f)$ with the corresponding matrix in $G L(\infty, \mathbb{Z})$.

Now we are able to present quite a reasonable generating set for Aut $F_{\infty}$

Theorem 5. Each automorphism in Aut $F_{\infty}$ is the composition of some IA-automorphism and some automorphism belonging to the subgroup generated by the lower triangular and the column-finite automorphisms, i.e., Aut $F_{\infty}=\left\langle\mathcal{T}^{-}, \mathcal{K}\right\rangle \cdot \mathcal{A}$.

Proof. First, we show that Aut $A_{\infty}=\left\langle\chi\left(\mathcal{T}^{-}\right), \chi(\mathcal{K})\right\rangle$. Let $A \in G L(\infty, \mathbb{Z})$. This means that in each row of $A$ all but a finite number of elements are zero. Since $\mathbb{Z}$ is a principal ideal domain, the invertibility of $A$ implies that the ideal generated by the elements of any row of $A$ is $\mathbb{Z}$. Therefore, there exists an elementary finitary matrix $B_{1}$ such that the product $A \cdot B_{1}$ has only one nonzero element in the first row. By a similar argument, there exists an elementary finitary matrix $B_{2}$ such that the product $A \cdot B_{1} \cdot B_{2}$ has only one nonzero element in the second row and this element stands below one of the zeros in the first row. We continue in this way, obtaining the infinite product $A \cdot\left(\prod_{i=1}^{\infty} B_{i}\right)$. This product is well defined [10], because for every natural $k$ and every natural $n \geq k$, in each finite product $A \cdot B_{1} \cdots B_{n}$ the first $k$ columns are the same and they are stabilized in $A \cdot B_{1} \cdots B_{k}$.

Hence, the matrix $A \cdot\left(\prod_{i=1}^{\infty} B_{i}\right)$ can be transformed (by a suitably chosen permutation matrix) to a lower triangular matrix $T$; more precisely, $A \cdot\left(\prod_{i=1}^{\infty} B_{i}\right) \cdot P=T$. Since $\left(\prod_{i=1}^{\infty} B_{i}\right)^{-1}=\prod_{i=1}^{\infty} B_{i}^{-1}$, we have $A=T \cdot P^{t} \cdot\left(\prod_{i=1}^{\infty} B_{i}^{-1}\right)$. Moreover, by construction we have $\prod_{i=1}^{\infty} B_{i}^{-1} \in \chi(\mathcal{K})$ and $P^{t} \in \chi(\mathcal{H})$, whence Aut $A_{\infty}=\left\langle\chi\left(\mathcal{T}^{-}\right), \chi(\mathcal{K})\right\rangle$, as required. Now, since $\mathcal{A}$ is the kernel of $\chi$, we can deduce the statement from the properties of the inverse image of the epimorphism $\chi$, and the result follows.

In fact, the proof of the above theorem implies the following statement.

Theorem 6. The group $G L(\infty, \mathbb{Z})$ is generated by the set of all lower triangular and all block-diagonal matrices.

For a generalization of this result from $\mathbb{Z}$ to an arbitrary associative ring, we refer the reader to [18]. It would also be of interest to know whether and how the results and methods described in [13-16] can be extended to Aut $F_{\infty}$.

Here we note that Theorem 6 can be proved also as follows. Swan proved (see [8]) that Aut $A_{\infty}$ is generated by the triangular automorphisms, where the word triangular is meant with respect to bases consisting of a fixed set of elements each, but (possibly) in a different order. Since every ordering of this set corresponds to some permutational automorphism, which in its turn is a product of strings [27], the result follows.

We denote by $\operatorname{Inn} F_{\infty}$ the group of inner automorphisms of $F_{\infty}$.

Proposition 4. a) The group $\mathcal{K}$ contains $\mathcal{H}$.

b) The group $\left\langle\mathcal{T}^{-}, \mathcal{H}\right\rangle$ contains $\operatorname{Inn} F_{\infty}$.

Proof. a) By induction on the number of strings, it can be shown that, in any finite product $\alpha$ of strings, every symbol $x_{i}$ can occur only in finitely many words $\alpha\left(x_{j}\right)$. Moreover, every string belongs to $\mathcal{K}$, so that $\mathcal{H} \subset \mathcal{K}$. 
b) Let $\alpha \in \operatorname{Inn} F_{\infty}$. Then for some $g \in$ Aut $F_{\infty}$ we have $\alpha(x)=g^{-1} x g$. Let $n$ be the minimal number for which $g \in\left\langle x_{1}, \ldots, x_{n}\right\rangle$. We define two automorphisms:

$\alpha_{1}\left(x_{1}\right)=g^{-1} x_{1} g, \ldots, \alpha_{1}\left(x_{n}\right)=g^{-1} x_{n} g, \alpha_{1}\left(x_{k}\right)=x_{k}$ for all $k>n$,

$\alpha_{2}\left(x_{1}\right)=x_{1}, \ldots, \alpha_{2}\left(x_{n}\right)=x_{n}, \alpha_{2}\left(x_{k}\right)=g^{-1} x_{k} g$ for all $k>n$.

Clearly $\alpha_{1} \in \mathcal{H}, \alpha_{2} \in \mathcal{T}^{-}$, and $\alpha=\alpha_{1} \circ \alpha_{2}$, which implies the statement. We note that $\alpha \neq \alpha_{2} \circ \alpha_{1}$.

Example. Let $\alpha_{3}\left(x_{1}\right)=x_{1}, \alpha_{3}\left(x_{2}\right)=x_{2}$, and let $\alpha_{3}\left(x_{i}\right)=\left[x_{1}, x_{2}\right] x_{i}$ for all $i>2$. It can be checked that the mapping $\alpha_{3}$ is an $I A$-automorphism that does not belong to $\mathcal{K}$.

We present a partial poset diagram for the subgroups of Aut $F_{\infty}$ considered in this paper:

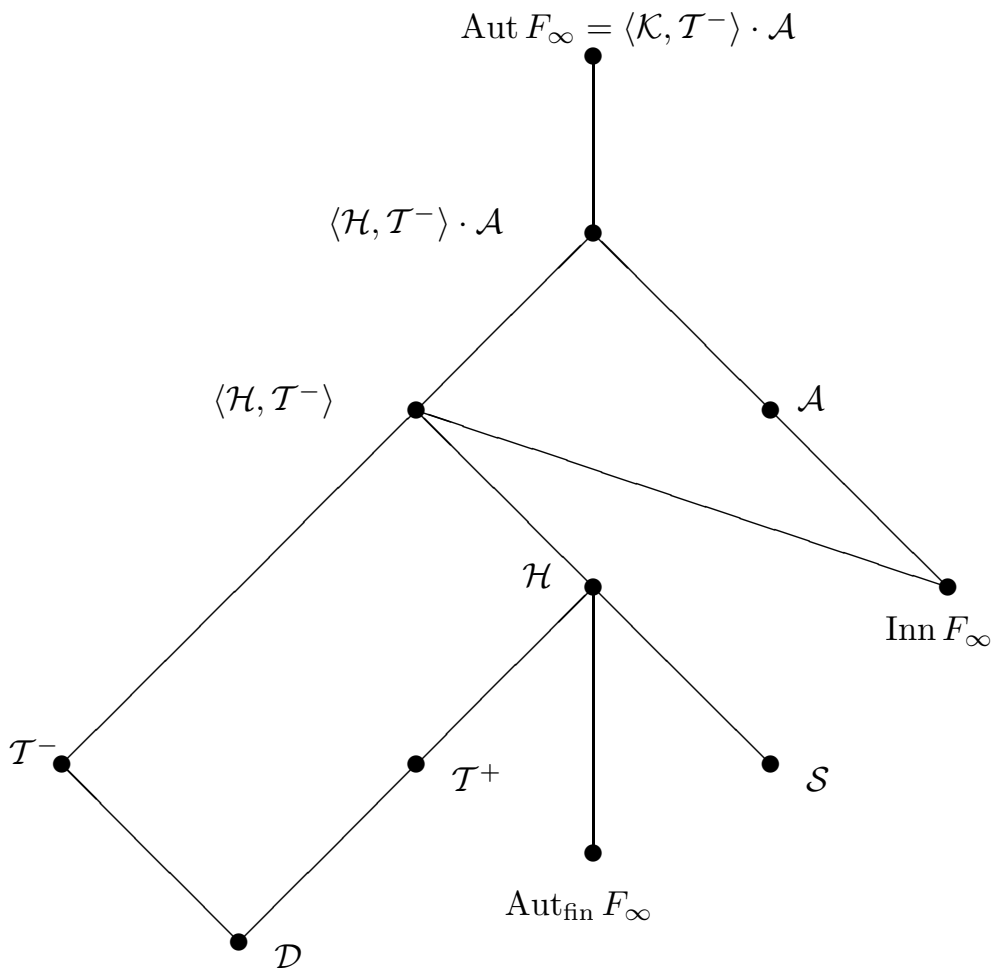

Using the properties of the epimorphism $\chi$ and the results of [5] and [12], we can describe some normal subgroups of Aut $F_{\infty}$.

Theorem 7. The group Aut $F_{\infty}$ contains two infinite countable families: one of maximal normal subgroups and the other of normal incomparable subgroups.

Proof. Since $\chi$ is an epimorphism, the maximal and normal subgroups in Aut $A_{\infty}$ are lifted by $\chi$ to maximal and normal subgroups in Aut $F_{\infty}$. Now, the first part of the statement follows from Corollary 0.7 of [5], and the second from [12] (see Theorem 7.3 and the discussion after its proof therein).

\section{§5. Results FOR SOME OTHER VARIETIES}

Now we turn to certain relatively free groups. Let $\mathfrak{V}$ be a variety, let $\mathcal{V}$ be the corresponding verbal subgroup, and let $F_{\infty} / \mathcal{V}$ be the relatively free group of $\mathfrak{V}$ of infinite 
countable rank on free generators $y_{1}, y_{2}, \ldots$ (see [23]). The natural homomorphism $F_{\infty} \rightarrow$ $F_{\infty} / \mathcal{V}$ induces a homomorphism

$$
\chi_{\mathfrak{V}}: \operatorname{Aut} F_{\infty} \rightarrow \operatorname{Aut}\left(F_{\infty} / \mathcal{V}\right)
$$

its kernel will be denoted by $\mathcal{A}_{\mathfrak{V}}$. Let $\mathfrak{A}, \mathfrak{A}_{n}$, and $\mathfrak{N}_{c}$ denote the varieties of Abelian groups, Abelian groups of exponent $n$, and nilpotent groups of class $c$, respectively. The following theorem summarizes the results on the surjectivity of $\chi_{\mathfrak{V}}$ obtained by many authors.

Theorem 8. The homomorphism $\chi_{\mathfrak{V}}: \operatorname{Aut} F_{\infty} \rightarrow \operatorname{Aut}\left(F_{\infty} / \mathcal{V}\right)$ is surjective if one of the following conditions is satisfied:

(1) $F_{\infty} / \mathcal{V}$ is nilpotent (see [4]);

(2) $\mathfrak{V}=\mathfrak{A}_{k} \mathfrak{A}_{l}$ for some natural numbers $k$ and $l$ (see [2]);

(3) $\mathfrak{A} \mathfrak{A} \subseteq \mathfrak{V} \subseteq \mathfrak{N}_{c} \mathfrak{A}$ for some natural number c (see [3, Theorem 2]).

If in the definition of the subgroups $\mathcal{T}^{+}, \mathcal{T}^{-}, \mathcal{K}$, and $\mathcal{H}$ we replace the generators $x_{i}$ by $y_{i}$, we obtain analogs of these subgroups in $\mathfrak{V}$, which we denote by $\mathcal{T}_{\mathfrak{V}}^{+}, \mathcal{T}_{\mathfrak{V}}^{-}, \mathcal{K}_{\mathfrak{V}}$, and $\mathcal{H}_{\mathfrak{V}}$, respectively.

The surjectivity of $\chi_{\mathfrak{V}}$ implies that the results of the preceding sections can easily be stated for the varieties occurring in Theorem 8.

Theorem 9. Let $\mathfrak{V}$ be any of the varieties listed in Theorem 8. Then:

a) $\mathcal{H}_{\mathfrak{V}}^{b} \supseteq\left\langle\mathcal{T}_{\mathfrak{V}}^{+}, \mathcal{S}\right\rangle$;

b) $\operatorname{Aut}\left(F_{\infty} / \mathcal{V}\right)=\left\langle\mathcal{T}_{\mathfrak{V}}^{-}, \mathcal{K}_{\mathfrak{V}}\right\rangle \cdot \mathcal{A}_{\mathfrak{V}}$

c) the group $\mathcal{K}_{\mathfrak{V}}$ contains $\mathcal{H}_{\mathfrak{V}}$;

d) the group $\left\langle\mathcal{T}_{\mathfrak{V}}^{-}, \mathcal{H}_{\mathfrak{V}}\right\rangle$ contains $\operatorname{Inn}\left(F_{\infty} / \mathcal{V}\right)$.

\section{REFERENCES}

[1] R. M. Bryant and D. M. Evans, The small index property for free groups and relatively free groups, J. London Math. Soc. (2) 55 (1997), 363-369. MR1438640 (97m:20047)

[2] R. M. Bryant and J. R. J. Groves, Automorphisms of free metabelian groups of infinite rank, Comm. Algebra 20 (1992), 783-814. MR1153050 (93f:20039)

[3] R. M. Bryant and C. K. Gupta, Automorphisms of free nilpotent-by-abelian groups, Math. Proc. Cambridge Philos. Soc. 114 (1993), 143-147. MR1219921 (94f:20052)

[4] R. M. Bryant and O. Macedońska, Automorphisms of relatively free nilpotent groups of infinite rank, J. Algebra 121 (1989), 388-398. MR0992773 (90e:20028)

[5] R. G. Burns and I. H. Farouqi, Maximal normal subgroups of the integral linear group of countable degree, Bull. Austral. Math. Soc. 15 (1976), 439-451. MR0430098 (55:3105)

[6] R. G. Burns and Lian Pi, Generators for the bounded automorphisms of infinite-rank free nilpotent groups, Bull. Austral. Math. Soc. 40 (1989), 175-187. MR1012826 (90i:20033)

[7] B. Chandler and W. Magnus, The history of combinatorial group theory. A case study in the history of ideas, Stud. Hist. Math. Phys. Sci., vol. 9, Springer-Verlag, New York, 1982. MR0680777 (85c:01001)

[8] J. M. Cohen, Aspherical 2-complexes, J. Pure Appl. Algebra 12 (1978), 101-110. MR0500902 (58:18405)

[9] R. Cohen, Classes of automorphisms of free groups of infinite rank, Trans. Amer. Math. Soc. 177 (1973), 99-120. MR0316581 (47:5128)

[10] R. G. Cooke, Infinite matrices and sequence spaces, MacMillan and Co., Ltd., London, 1950. MR0040451 (12:694d)

[11] J. D. Dixon and B. Mortimer, Permutation groups, Grad. Texts in Math., vol. 163, Springer-Verlag, New York, 1996. MR1409812 (98m:20003)

[12] I. H. Farouqi, On an infinite integral linear group, Bull. Austral. Math. Soc. 4 (1971), 321-342. MR0280608 (43:6327)

[13] W. Hołubowski, Subgroups of infinite triangular matrices containing diagonal matrices, Publ. Math. Debrecen 59 (2001), no. 1-2, 45-50. MR1853490

[14] _ Parabolic subgroups of Vershik-Kerov's group, Proc. Amer. Math. Soc. 130 (2002), 25792582. MR1900864 (2003b:20074) 
[15] _ Most finitely generated subgroups of infinite unitriangular matrices are free, Bull. Austral. Math. Soc. 66 (2002), 419-423. MR1939204 (2003i:20092)

[16] Free subgroups of the group of infinite unitriangular matrices, Internat. J. Algebra Comput. 13 (2003), 81-86. MR1970869 (2004c:20090)

[17] , A new measure of growth for groups and algebras (submitted).

[18] W. Hołubowski and N. A. Vavilov, Infinite dimensional general linear groups (in preparation).

[19] R. C. Lyndon and P. E. Schupp, Combinatorial group theory, Ergeb. Math. Grenzgeb., Bd. 89, Springer-Verlag, Berlin-New York, 1977. MR0577064 (58:28182)

[20] O. Macedońska-Nosalska, Note on automorphisms of a free abelian group, Canad. Math. Bull. 23 (1980), 111-113. MR0573567 (81h:20032)

[21] , The abelian case of Solitar's conjecture on infinite Nielsen transformations, Canad. Math. Bull. 28 (1985), 223-230. MR0782780 (86j:20025)

[22] W. Magnus, A. Karrass, and D. Solitar, Combinatorial group theory. Presentations of groups in terms of generators and relations, Intersci. Publ., New York etc., 1966. MR0207802 (34:7617)

[23] H. Neumann, Varieties of groups, Springer-Verlag New York, Inc., New York, 1967. MR0215899 $(35: 6734)$

[24] J. Nielsen, Die Isomorphismengruppe der freien Gruppen, Math. Ann. 91 (1924), 169-209. MR1512188

[25] J. D. Sharp and S. Thomas, Uniformization problems and the cofinality of the infinite symmetric group, Notre Dame J. Formal Logic 35 (1994), no. 3, 328-345. MR1326117 (96g:20001)

[26] W. Sierpiński, Sur une décomposition d'ensembles, Monatsh. Math. Phys. 35 (1928), $239-248$. MR1549531

[27] S. Thomas, The cofinalities of the infinite-dimensional classical groups, J. Algebra 179 (1996), no. 3, 704-719. MR1371739 (96k:20005)

Department of Mathematics, University of Manitoba, Winnipeg, Manitoba, R3T 2 N2 CanADA

Institute of Mathematics, Silesian University of Technology, Kaszubska, 23, 44-100 GliWICE, POLAND

E-mail address: w.holubowski@polsl.pl

Received 19/JUN/2006

Originally published in English 